Z Rheumatol 2009 $68: 795-796$

DOI 10.1007/s00393-009-0554-z

Online publiziert: 25. November 2009

(c) Springer Medizin Verlag 2009

\author{
E. Genth ${ }^{1} \cdot$ W. Rüther ${ }^{2}$ \\ ${ }^{1}$ Rheumaklinik und Rheumaforschungsinstitut, Aachen \\ ${ }^{2}$ Klinik für Orthopädie, Universitätsklinikum Eppendorf, \\ Hamburg und Klinikum Bad Bramstedt
}

\title{
Konservative Therapie der Arthrose zwischen Evidenz, Konsens und Nonsens
}

Liebe Kolleginnen, liebe Kollegen,

es gibt wenige therapeutische Bereiche, in denen eine umfassende und ausgewogene Bewertung der vorhandenen therapeutischen Evidenz so notwendig ist wie bei der Osteoarthrose. Die verschiedenen Formen der Arthrose stellen die größte Gruppe muskuloskelettaler Krankheiten dar mit erheblichen Auswirkungen auf die funktionale Gesundheit und hohen Kosten für das Gesundheitssystem und die Gesellschaft. Die Prävalenz sowohl der symptomatischen wie auch der radiologisch nachgewiesenen Hüftarthrose ist niedriger als die der Kniearthrose und variiert in Abhängigkeit von Alter, Geschlecht, Region und anderen Faktoren. Bis heute existiert keine krankheitsmodifizierende Therapie. Umso vielfältiger sind die symptomatischen und funktionellen Behandlungsformen und -strategien mit einem blühenden Markt von Verfahren mit nachgewiesener oder nicht nachgewiesener Wirksamkeit und nachgewiesener Unwirksamkeit. Selbst unter Experten werden relevante Unterschiede auf der Ebene der Metaanalyse und Interpretation deutlich, die auch in Unterschieden der Empfehlungen und Leitlinien deutlich werden.

\section{Bis heute existiert keine krankheitsmodifizierende Arthrosetherapie}

Die OARSI („Osteoarthritis Research Society International“) hat 2007 Empfeh- lungen zum Management der Hüft- und Kniearthrose veröffentlicht [10], die auf einer kritischen Bewertung von 23 Leitlinien und systematischen Reviews derzeitiger wissenschaftlicher Evidenz beruhen. Überwiegend gingen in die Bewertung Leitlinien zur Gonarthrose oder zur Arthrose der Hüft- und Kniegelenke ein. Von den 51 bewerteten Behandlungsmodalitäten wurden 20 empfohlen.

Keines der Verfahren erreichte in Bezug auf Schmerzlinderung eine hohe Effektstärke (ES >0,8). Moderate Effektstärken von 0,52 wurden für aerobe Übungsbehandlungen ermittelt, die entsprechenden Werte für Paracetamol und nichtsteroidale Antirheumatika (NSAR) lagen mit 0,21 und 0,32 deutlich niedriger. Für aerobe Übungsbehandlungen, Krafttraining und Übungen im Wasser ergab sich die höchste Evidenz und entsprechend der höchste Empfehlungsgrad, des Weiteren auch für Patientenschulung, Selbstmanagementtraining, Paracetamol und NSAR.

Die Effektstärken in Bezug auf eine Verbesserung der Funktion durch nichtmedikamentöse Interventionen waren ebenfalls gering. Allenfalls die Gewichtsreduktion zeigte eine deutliche Besserung der Funktionswerte (ES=0,69), nicht jedoch der Schmerzminderung.

Für die Arthrose der Hand wurden 2007 die aktualisierten Empfehlungen der EULAR publiziert [9], an denen Experten aus 15 Ländern Europas beteiligt waren. Es wurden 11 Kernvorschläge mit 17 Behandlungsmodalitäten in 3 DelphiRunden zu allgemeinen Vorgehenswei- sen, medikamentösen und nichtmedikamentösen Behandlungsmodalitäten und zur chirurgischen Behandlung formuliert. Die allgemeinen Empfehlungen beruhen auf Expertenkonsensus. Patienteninformation und -schulung und Übungsbehandlung, Schienenversorgung, systemisch und lokal applizierte NSAR-Therapie hatten moderate Effektstärken in Bezug auf Schmerzlinderung, NSAR in geringem Maße auch auf die Funktion.

Gelenkschmerzen, insbesondere
unter Belastung und Bewegung, sind
das führende Symptom der Arthrose.

In der überwiegenden Zahl von Patienten mit Knie-, Hüft- oder Fingerpolyarthrose ist der Zusammenhang zwischen lokaler Gelenkschädigung und in erster Linie Nocizeptor-induziertem Schmerz unter Bewegung oder Belastung deutlich. Für einen beträchtlichen Teil der Arthrosepatienten ist die geringe Übereinstimmung von Schmerzintensität mit dem Grad der strukturellen Schädigung dokumentiert. 30-40\% der Patienten mit den radiologischen Schädigungsgraden III und IV an den Hüft- oder Kniegelenken geben keine Schmerzen an, und etwa 10\% der Patienten mit mäßigen bis starken Knieschmerzen haben normale Röntgenbefunde an den Gelenken [3]. Psychologische Faktoren wie Angst und Depression [2] sowie zentrale Schmerzsensibilisierung (Hyperalgesie, Allodynie), moduliert durch genetische Faktoren [7], spielen eine wesentliche Rolle. Dies bedeutet, dass 
ein individuell unterschiedlicher Teil der Symptombildung außerhalb des Gelenks, nämlich im zentralen Nervensystem stattfindet und unterschiedliche Schmerzmechanismen beteiligt sein können.

Bei der Behandlung von Arthroseschmerzen, aber auch für funktionelle Therapieeffekte, spielen Placebowirkungen eine wesentliche Rolle. Dies wurde erneut deutlich am Beispiel der GAIT- („Glucosamin/Chondroitin Arthritis Intervention Trial“-) Studie [1]. In dieser Studie wurden Patienten mit symptomatischer Kniearthrose in 5 Behandlungsgruppen randomisiert: Glucosamin, Chondroitinsulfat, Kombination von beidem, Celecoxib und Placebo. Primäres Ziel war eine Reduktion von Schmerzen um $20 \%$, gemessen mit dem WOMAC- („Western Ontario McMaster-") Score. Dieses Ziel wurde bei $64 \%$ der Glucosamin-Gruppe, 65\% der Chondroitinsulfat-Gruppe, $67 \%$ der Kombinationsgruppe, 79\% der Celecoxib-Gruppe und 6o\% der Placebogruppe erreicht.

Eine systematische Analyse von Placeboeffekten durch Zhang et al. [11] zeigte, dass dieses Ergebnis nicht die Ausnahme, sondern die Regel ist. Die im Vorher-Nachher-Vergleich bei 198 Studien ermittelten Effektstärken lagen bei unbehandelten Patienten bei O,03, bei den placebobehandelten Patienten bei o,51 und somit im Bereich moderater Effektstärken für Schmerzen, Steifigkeitsgefühl und Selbstbeurteilung der Funktion. Die Unterschiede zwischen Nichtbehandlung und Placebobehandung waren größer als die zwischen den Placebo- und Verumgruppen. Allerdings sind Placeboeffekte nur bei kontinuierlichen und nicht bei binären Merkmalen nachweisbar [4]. Auffällig ist, dass die Effektstärke des Placeboeffekts in Bezug auf Schmerzen bei der Fingerpolyarthrose mit o,8 deutlich höher ist als bei der Arthrose des Hüftgelenks. Bemerkenswert ist auch, dass entsprechende Effektstärken auch für die Selbstbewertung von Funktion $(0,49)$ und Steifigkeitsgefühl $(0,43)$ bestanden und sogar für das Arztgesamturteil (o,66). Bei der kleinen Zahl von Studien, die gleichzeitig eine Fremdbeurteilung aufgrund von Messungen durchführten, waren die Effektstärken deutlich niedriger.

Für die Stärke des Placeboeffekts ausschlaggebend sind die Kontextfaktoren der Behandlung [8]. Diese liegen insbesondere in der persönlichen Interaktion von Behandler und Behandeltem und in Bedingungen der Behandlungssituation. Darüber hinaus konnten NeuroimagingStudien mit funktioneller Magnetresonanztomographie des Gehirns [5] zeigen, dass Placeboeffekte mit Veränderungen der Aktivität verschiedener Hirnareale einhergingen und Dopamin- und OpioidRezeptor-abhängige Effekte [12] eine wichtige Rolle spielen.

Die Bedingungen von Nichtbehandlung und von Vorbehandlung sind im Vergleich zu festgelegten Bedingungen von Behandlung nicht immer einfach zu definieren. Auch die Verblindung von Behandelten und Behandlern kann je nach Behandlungsverfahren schwierig sein. Placebo- oder Scheinbehandlungen sind bei Krankengymnastik oder operativen Therapien schwierig zu ,inszenieren“. Scheinoperationen können jedoch, wie Mosely et al. [6] am Beispiel einer arthroskopischen Behandlung der Gonarthrose zeigte, eindrucksvolle Placeboeffekte aufweisen.

\section{$\checkmark$ Evidenz und Expertenkonsens sind die wesentliche Basis der Bewertung von Wirksamkeit in der Arthrosetherapie}

Die Beiträge in diesem Heft behandeln Aspekte der operativen Therapie und Hilfsmittelversorgung insbesondere der Kniearthrose, bewerten Nutzen und Risiken von sportlicher Aktivität bei Arthrose und geben eine kritische Bewertung. Evidenz und Expertenkonsens sind die wesentliche Basis der Bewertung von Wirksamkeit in der Arthrosetherapie. Die adäquate Kontrolle von Placeboeffekten bleibt eine Herausforderung der wissenschaftlichen Evaluation.

Ihre

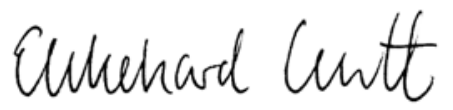

E. Genth

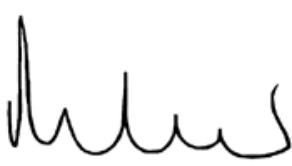

W. Rüther

\section{Korrespondenzadresse}

Prof. Dr. E. Genth

Rheumaklinik und Rheumaforschungsinstitut Burscheider Markt 24, 52066 Aachen

mail@ekkehard-genth.de

Interessenkonflikt. Der korrespondierende Autor gibt an, dass kein Interessenkonflikt besteht.

\section{Literatur}

1. Clegg DO, Reda DJ, Harris CL et al (2006) Glucosamine, chondroitin sulfate, and the two in combination for painful knee osteoarthritis. N Engl J Med 354:795-808

2. Creamer P, Hochberg MC (1998) The relationship between psychosocial variables and pain reporting in osteoarthritis of the knee. Arthritis Care Res 11:60-65

3. Hannan MT, Felson DT, Pincus T (2000) Analysis of the discordance between radiographic changes and knee pain in osteoarthritis of the knee. J Rheumatol 27:1513-1517

4. Hrobjartsson A, Gotzsche PC (2004) Is the placebo powerless? Update of a systematic review with 52 new randomized trials comparing placebo with no treatment. J Intern Med 256:91-100

5. Kong J, Gollub RL, Rosman IS et al (2006) Brain activity associated with expectancy-enhanced placebo analgesia as measured by functional magnetic resonance imaging. J Neurosci 26:381-388

6. Moseley JB, O'Malley K, Petersen NJ et al (2002) A controlled trial of arthroscopic surgery for osteoarthritis of the knee. N Engl J Med 347:81-88

7. van Meurs JB, Uitterlinden AG, Stolk L et al (2009) A functional polymorphism in the catechol-O-methyltransferase gene is associated with osteoarthritis-related pain. Arthritis Rheum 60:628-629

8. Wampold BE, Imel ZE, Minami T (2007) The story of placebo effects in medicine: evidence in context. J Clin Psychol 63:379-390

9. Zhang W, Doherty M, Leeb BF et al (2007) EULAR evidence based recommendations for the management of hand osteoarthritis: report of a Task Force of the EULAR Standing Committee for International Clinical Studies Including Therapeutics (ESCISIT). Ann Rheum Dis 66:377-388

10. Zhang W, Moskowitz RW, Nuki G et al (2007) OARSI recommendations for the management of hip and knee osteoarthritis, part l: critical appraisal of existing treatment guidelines and systematic review of current research evidence. Osteoarthritis Cartilage 15:981-1000

11. Zhang W, Robertson J, Jones AC et al (2008) The placebo effect and its determinants in osteoarthritis: meta-analysis of randomised controlled trials. Ann Rheum Dis 67:1716-1723

12. Zubieta JK, Stohler CS (2009) Neurobiological mechanisms of placebo responses. Ann NY Acad Sci 1156:198-210 